\title{
Le défi coopératiste du syndicat de la typographie lyonnaise
} (1864-1999)

\author{
El reto cooperativista del sindicato de la tipografía de Lyon
}

(1864-1999)

\section{The cooperativist challenge of the Lyonnais printers' union (1864-1999)}

\section{Claire Bonici}

Numéro 326, octobre 2012

La course à la taille

Concentration in the social and solidarity economy

URI : https://id.erudit.org/iderudit/1016871ar

DOI : https://doi.org/10.7202/1016871ar

Aller au sommaire du numéro

Éditeur(s)

Association Recma

ISSN

1626-1682 (imprimé)

2261-2599 (numérique)

Découvrir la revue

Citer cet article

Bonici, C. (2012). Le défi coopératiste du syndicat de la typographie lyonnaise

(1864-1999). Revue internationale de l'économie sociale, (326), 90-99.

https://doi.org/10.7202/1016871ar
Résumé de l'article

Souvent perçue comme une alternative innovante aux dérives capitalistes, l'économie sociale et solidaire puise ses origines dans d'anciennes traditions d'entraide, renouvelées au $\mathrm{XIX}^{\mathrm{e}}$ siècle par l'émergence de groupements constitués en réaction au libéralisme post-révolutionnaire (associations, mutuelles, coopératives, syndicats). Face à l'attrait actuel du concept, il semble opportun de comprendre le contexte d'un développement qui s'inscrit dans un besoin d'organiser des solidarités. Né en mars 1864, l'exemple des ouvriers typographes lyonnais offre une approche intéressante du mouvement coopératif, en raison de son caractère précurseur et de sa longévité dans une corporation fortement structurée. Il est en outre révélateur des défis auxquels la coopération fut confrontée et de l'ambiguité de ses rapports avec le mouvement syndical. Dans un environnement capitaliste concurrentiel, l'expérience témoigne d'un siècle de pratiques coopératives, durant lequel espoirs et idéaux initiaux de fonctionnement démocratique ont été confrontés à des difficultés de gouvernance et à la nécessité de trouver, sans cesse, un équilibre entre le respect de l'esprit militant et la maîtrise économique de la structure. 


\title{
LE DÉFI COOPÉRATISTE DU SYNDICAT DE LA TYPOGRAPHIE LYONNAISE (1864-1999)
}

\author{
par Claire Bonici*
}

* Docteure en droit. Mél.: claire. bonici@dbmail.com.
Souvent perçue comme une alternative innovante aux dérives capitalistes, l'économie sociale et solidaire puise ses origines dans d'anciennes traditions d'entraide, renouvelées au XIX $\mathrm{X}^{e}$ siècle par l'émergence de groupements constitués en réaction au libéralisme post-révolutionnaire (associations, mutuelles, coopératives, syndicats). Face à l'attrait actuel du concept, il semble opportun de comprendre le contexte d'un développement qui s'inscrit dans un besoin d'organiser des solidarités. Né en mars 1864, l'exemple des ouvriers typographes lyonnais offre une approche intéressante du mouvement coopératif, en raison de son caractère précurseur et de sa longévité dans une corporation fortement structurée. Il est en outre révélateur des défis auxquels la coopération fut confrontée et de l'ambiguïté de ses rapports avec le mouvement syndical. Dans un environnement capitaliste concurrentiel, l'expérience témoigne d'un siècle de pratiques coopératives, durant lequel espoirs et idéaux initiaux de fonctionnement démocratique ont été confrontés à des difficultés de gouvernance et à la nécessité de trouver, sans cesse, un équilibre entre le respect de l'esprit militant et la maîtrise économique de la structure.
(1) L'ONU a décrété 2012 Année internationale des coopératives (communiqué de presse, 21 décembre 2009, DEV/2784) En France, l'économie sociale et solidaire (ESS) est à l'origine d'initiatives citoyennes diverses relayées par les pouvoirs publics, notamment avec la création, dans le gouvernement Ayrault, d'un ministre délégué à l'Economie sociale et solidaire auprès du ministre de l'Economie et des Finances.
D epuis 2008, l'amplification de la crise s'accompagne d'une dénonciation de plus en plus sévère d'un modèle de société incompris et inféodé à la sphère financière. Dans le même temps, la recherche d'alternatives se développe. Elle renouvelle une réflexion fondée sur la nécessité de repenser notre système, pour remettre l'économie au service de l'humain, et suscite ainsi un regain d'intérêt pour le concept d'économie sociale et solidaire, qui peinait à se perpétuer dans le capitalisme et à s'imposer dans l'espace médiatique ${ }^{(1)}$. Le fonctionnement démocratique de cette économie et sa dynamique de développement la font apparaître comme une force de transformation, une source de réflexion dans l'impasse économique à laquelle notre société semble confrontée.

Si l'économie sociale et solidaire incarne aujourd'hui une vision différente d'entreprendre, elle n'est pas une réalité nouvelle. Elle inscrit ses origines dans une filiation marquée par la diversité et la pluralité des plus anciennes formes d'associations humaines (Gueslin, 1987). Mais c'est avec le développement de la société industrielle, au XIX ${ }^{\mathrm{e}}$ siècle, que ses pratiques se sont véritablement modernisées, pour réagir contre les excès d'un libéralisme non régulé et souvent source de dénuement pour 
la population laborieuse. Le mouvement de remise en question de l'économie comme source de progrès social, qui va progresser alors, n'est pas sans évoquer le contexte actuel.

Face au renouveau de l'économie sociale et solidaire, il parait utile de porter un regard sur le passé pour mieux comprendre un concept encore méconnu tant dans son mode d'organisation que dans son rôle dans l'appareil productif. Le but de cette contribution consistera à s'arrêter sur quelques-uns des aspects du mouvement coopératif à travers le prisme des ouvriers du livre à Lyon, une corporation engagée dans l'expérience coopératiste depuis 1864 et jusqu'à aujourd'hui. Cet exemple permettra, en outre, de s'intéresser aux origines, au développement du coopératisme de production au sein du mouvement syndical, sans méconnaittre la complexité de leurs rapports.

\section{Un contexte favorable: Lyon, creuset de " culture " ouvrière}

(2) En 1806 le premier conseil de prud'hommes, mais aussi dès les années 1830 les sociétés de secours mutuels, les coopératives de consommation, puis les syndicats professionnels.
Haut lieu industriel, l'agglomération lyonnaise s'est développée autour de La Fabrique, une activité de transformation de la soie marquée par le modèle de la manufacture dispersée avec des chefs d'atelier propriétaires de leurs outils et fiers de leur savoir-faire. Ce modèle d'industrie sans industriels différenciait la ville des autres grandes métropoles industrielles comparables, où la production avait lieu dans de grandes unités avec des produits standardisés (Frobert, 2007). Après la Révolution, ce système de production favorise l'invention de modes de régulation développés en commun ${ }^{(2)}$ et de nouvelles formes d'organisation, ainsi que la résurgence de réflexes de solidarité hérités de l'Ancien Régime. Il contribue à l'épanouissement d'une remarquable conscience sociale ouvrière en réaction à l'élite locale fortunée (Moissonnier, 1984; Sheridan, 1981; Rude, 2001). Ces particularismes font de la cité lyonnaise un lieu privilégié, fertile (Rude, 1977) pour les doctrines qui remettent en cause la société et prônent la transformation du système existant sur des bases nouvelles, dans une perspective de justice et de solidarité. Le saint-simonisme fait dans la ville de nombreux adeptes dont des notables, tel Arles-Dufour, qui vont influencer la politique locale. Fourier comme Proudhon vivent dans la cité, partagent la vie des ouvriers et nourrissent leurs réflexions de leurs observations des antagonismes entre classes sociales. Lyon, théâtre des batailles des canuts de 1831, devient un point de convergence pour des penseurs comme Flora Tristan ou Cabet. Toutes ces doctrines s'appuient sur des groupements intermédiaires et des mécanismes de régulation collective pour mettre en place des dispositifs de protection de solidarité. Dans ce contexte, l'idée de mutuellisme et celle de coopération ouvrière rencontrent rapidement un vif succès. Le plus souvent clandestines, ces initiatives font l'objet d'une étroite surveillance de la part de pouvoirs publics suspicieux, comme le montrent les rapports des parquets et des commissaires de 1849 à 1851 (Tchernoff, 1905). 
Dès les années 1830-1840, les premières expériences d'« économie alternative " voient le jour, influencées par les théories fouriéristes connues par nombre d'ouvriers lyonnais dès 1833. Lyon occupe ainsi une place essentielle dans l'histoire de la coopération, et ce n'est pas fortuit si la première coopérative française de consommation est fondée à l'initiative d'un Lyonnais, Michel Derrion, un bourgeois séduit par les idées fouriéristes qui engagea sa fortune dans l'expérience du "commerce véridique et social ». L'élan associationniste officiel de 1848 amplifie ensuite le mouvement, particulièrement à Lyon, où il provoque l'éclosion d'associations pré-coopératives de consommation et de production. Les associations ouvrières apparaissent alors comme un moyen privilégié de transformation sociale, une solution alternative permettant aux travailleurs de gérer librement leur production, de s'approprier le fruit de leur ouvrage et de se soustraire aux dérives d'un système libéral concurrentiel générateur de misère. Cette "flambée associative" est néanmoins de courte durée: les mesures coercitives qui suivent le coup d'Etat du 2 décembre 1851 anesthésient ce mouvement coopératiste, jugé trop proche des idéaux socialistes et du mouvement républicain lyonnais. Ce n'est finalement qu'à partir de 1860 que le concept de coopération émerge réellement dans un "second printemps de l'association " (Lequin, 1977), initié dans un mouvement d'inflexion du régime impérial. Il se traduit, dans le domaine de la coopération, par la préparation d'une loi, ainsi que par le lancement d'une enquête sur les coopératives.

\section{Un projet de coopération audacieux pour des ouvriers u libres n: I'Association typographique lyonnaise}

A Lyon, les typographes furent une des premières corporations à fonder une association ouvrière de production: l'Association typographique lyonnaise. Cette première coopérative de la typographie lyonnaise, comme celles qui furent créées ultérieurement, illustre les défis inhérents à la double nature de la coopérative. Son histoire de plus d'un siècle montre la richesse de la formule pour la classe ouvrière, mais aussi la difficulté à trouver un équilibre entre respect de l'esprit militant et maitrise économique de la structure dans un environnement capitaliste concurrentiel. Dès mars 1864, une cinquantaine de membres du syndicat des typographes lyonnais décident de constituer un capital commun pour créer une coopérative de production. Les propos tenus au Congrès national des syndicats ouvriers de 1886 sont alors sans ambiguité: ils affirment la volonté des ouvriers de s'affranchir du joug patronal et de créer les moyens de leur émancipation sociale par la prise de possession de l'outil de production. Le projet est ambitieux compte tenu de la modicité des salaires et de l'importance des dépenses affectées au logement et à l'alimentation (environ $70 \%$ de la part du budget total). En outre, aucun cadre juridique particulier ne permet alors de créer une entreprise collective hors 
(3) La caisse annexe, ou Annexe, devient la Chambre syndicale typographique lyonnaise en 1883, puis la quatorzième section lorsque la Fédération du livre s'impose.

(4) Pour comparaison, la cotisation fixée pour la prestation chômage s'élève alors à 40 centimes (archives de l'institut régional CGT d'histoire sociale, Lyon, procès-verbal de la séance du 23 juin 1862 du comité).

(5) L'emprunt est possible grâce à I'intermédiaire de la Société lyonnaise de crédit au travail, coopérative de crédit fondée en 1866 sur le modèle de la banque associative de Jean-Pierre Beluze. Cette banque vise à être une caisse d'épargne et une société de crédit destinée à financer la création d'associations diverses. du schéma civiliste du contrat de société (Code civil, art. 1832 et sq.), qui implique le partage du bénéfice monétaire en proportion des apports financiers de chaque associé. Créé hors de toute norme légale, le projet "pré-coopératif " des typographes s'apparente donc, à l'origine, à une société de fait, dénommée Association typographique lyonnaise (ATL). Celle-ci fonctionne en empruntant les règles inadaptées du Code civil et du Code du commerce jusqu'à ce que la loi du 24 juillet 1867 relative au commerce en général donne, dans son titre 3 , un premier cadre légal aux associations ouvrières de production.

Le projet d'association coopérative est adopté au sein du syndicat le 3 mars 1864, lors d'une réunion du comité consultatif (3) de l'Annexe. L'annonce de la création est largement diffusée par une circulaire adressée aux sociétaires pour leur demander leur adhésion au projet. La proposition reçoit alors un accueil mitigé: début mai, moins du quart des syndiqués répondent favorablement à l'appel et commencent à verser la somme non négligeable ${ }^{(4)}$ de 1 franc par mois et par action de 100 francs inscrite. Fin 1865, l'apport n'est toujours pas suffisant, en dépit de l'augmentation du nombre d'adhérents intéressés et du capital réuni. Ainsi, lorsqu'en avril 1866 une imprimerie est mise en vente dans le cadre d'un départ dû à l'âge, à peine un huitième de la somme nécessaire est réuni. L'opération se concrétise néanmoins grâce à un emprunt à la caisse des associations populaires Walras et Cie de Paris ${ }^{(5)}$ et à la bienveillance du vendeur, qui consent à garder 150 des 320 actions que, faute de moyens financiers, les ouvriers ne peuvent envisager d'acquérir de façon immédiate.

\section{Un fonctionnement démocratique dans une période d'expansion du capitalisme libéral}

A partir de 1866, l'ATL fait fonctionner un atelier social ouvert aux ouvriers de la corporation des typographes, ainsi qu'à tout auxiliaire employé qui en fait la demande: lithographes, écrivains, dessinateurs, brocheurs, papetiers de toute nature (art. 7, \$2). Les statuts exigent toutefois que les impétrants soient majeurs, qu'ils offrent "des garanties de probité et de moralité et qu'ils s'engagent à faire donner à [leurs] enfants l'instruction élémentaire» (art. 7, \$1).

Sur le plan juridique, l'ATL préfigure certains des principes qui seront ensuite applicables aux sociétés coopératives, notamment le respect des règles démocratiques et la prévalence de l'intérêt général. Chacun des associés contribue aux choix des orientations de l'association selon le principe "Une personne égale une voix ». Pour les grandes décisions stratégiques, le pouvoir est exercé par l'assemblée générale: elle nomme pour le fonctionnement quotidien un conseil composé de huit membres dirigeants et pouvant déléguer ses pouvoirs à un directeur. La régulation s'effectue par un système de contrôle diligenté par trois commissaires. Les bénéfices sont principalement destinés aux actionnaires et à la constitution de fonds de réserve et de prévoyance pour assurer la pérennité financière de l'ATL. 
Malgré la ferveur de ses fondateurs, l'ATL reste pendant les premières années de son existence soumise aux aléas d'un contexte politique et juridique peu favorable au développement des associations ouvrières, notamment dans l'imprimerie. Il faut dire que le secteur fait alors l'objet de sévères restrictions et contrôles: les structures sont très dépendantes de leur dirigeant, seul détenteur du brevet nécessaire à l'exercice de la profession. La censure sévit et les imprimeurs sont victimes de nombreuses condamnations. En outre, les associations ouvrières naissantes, avec leurs particularités, doivent faire face à la concurrence de l'industrie privée dans une période de difficultés économiques et de profondes mutations technologiques. Les membres de l'ATL, préparés à cette dure concurrence de leurs confrères lyonnais, parviennent néanmoins à développer l'activité de leur coopérative, comme ils le feront jusqu'en 1995.

\section{La création de I'Imprimerie nouvelle lyonnaise}

Plus difficile à admettre est la concurrence générée par la création d'une seconde association coopérative typographique, à l'initiative du syndicat lui-même. Le contexte est alors modifié: l'heure est au renouveau de la coopérative de production suite à la vague de grèves des années 18791880 et aux congrès ouvriers de Paris, de Lyon et de Marseille. Dans le même temps, en 1881, lors du premier congrès typographique (au cours duquel la Fédération du livre est creée), le principe coopératif apparaît comme une question centrale des discussions. Les deux délégués lyonnais présents à la réunion parisienne interviennent, à leur retour, lors de la première assemblée générale du syndicat: ils pourfendent les patrons et enjoignent leurs confrères à devenir possesseurs de leur outil de travail et à fonder une imprimerie nouvelle pour avoir un salaire proportionnel à leurs besoins et mettre un terme aux grèves pour toujours. L'idée est plébiscitée et le comité adopte la résolution de fonder une société coopérative à capital variable sous le patronage du syndicat et d'acheter du matériel d'imprimerie. A cet effet, un prélèvement obligatoire et forfaitaire de 1 franc est effectué sur chacune de leurs cotisations. Lambition de combattre "sur le terrain économique les patrons " est impulsée par la fédération. Le directeur de l'ATL proteste contre cette décision en faisant valoir la concurrence déloyale générée et l'urgence de s'unir contre le patronat plutôt que de se diviser. Ces arguments n'ont pas plus de résultat que les objections statutaires, notamment celle selon laquelle le comité n'aurait pas eu le droit de disposer de fonds initialement non versés pour la création d'une association coopérative.

La volonté du syndicat est alors ferme: il entend promouvoir un type d'association plus combatif, plus " collectiviste" que l'ATL. La nouvelle coopérative est conçue comme une "œuvre commune ": il ne s'agit pas de susciter une adhésion librement consentie, mais d'impliquer tous les syndiqués, qui deviennent d'office associés et s'engagent dans la lutte 
menée par la classe ouvrière pour échapper au salariat imposé par le patronat. A l'origine de cette démarche, la fédération contribue largement à son financement par un prêt d'un montant cinq fois supérieur au modeste apport de la section (1000 francs), ce qui permet l'achat, après faillite, d'un matériel d'imprimerie. Cette entreprise est toutefois compromise, car au moment de l'achat le président du comité disparaît en dérobant une partie de la somme. Il faut alors toute la détermination des syndiqués pour finaliser la transaction et réunir la somme grâce à une décision de prélèvement d'impôt sur les salaires des 200 syndiqués et à une suppression des primes trimestrielles.

Le 12 novembre 1883, les statuts de l'Imprimerie nouvelle lyonnaise (INL) sont adoptés, après échec des négociations pour le rachat des actions par l'ATL, les membres de cette dernière ayant préféré conserver leurs spécificités. L'acte fondateur de la coopérative est significatif de l'étroitesse des liens entre le syndicat et la coopérative, délibérément envisagée comme un moyen au service de l'action syndicale: l'ingérence est palpable dans l'imbrication financière des deux structures (syndicat et INL), dans l'octroi de la qualité d'associé à tout syndiqué et dans la gouvernance de l'association. Ainsi, l'article 12 des statuts prévoit que les difficultés relatives au prix de la main-d'œuvre sont soumises à l'arbitrage du syndicat. L'atelier social initialement destiné à accueillir l'ensemble des associés reste réservé à un nombre restreint, faute d'ouvrage suffisant: un système de tirage au sort permet de déterminer les personnes, au gré des commandes.

La création de l'INL se situe dans un contexte différent pour les associations ouvrières, désormais appelées sociétés coopératives. A partir des années 1870, le concept évolue au sein du mouvement ouvrier français en voie de formation. Il devient l'une des questions centrales des trois premiers congrès ouvriers: en dépit de nombreuses controverses, le principe coopératif se développe. Dans le même temps, le législateur profite de la loi du 24 juillet 1864 pour insérer un titre spécial pour ce type d'association, auquel les dispositions du Code de commerce ne peuvent convenir. La loi consacre en particulier le principe de variabilité, plus adapté aux associations ouvrières de production, où le capital s'accroît progressivement dans un contexte de personnes "nomades" (art. 48) [Pierre de Vellefrey, 1875]. Pour se conformer aux prescriptions légales, les fondateurs utilisent un artifice juridique: les actions de l'INL sont attribuées de façon nominative aux syndiqués, puisque le syndicat ne dispose pas encore d'une personnalité juridique. Il est toutefois convenu de manière implicite que ces derniers renoncent tacitement à la propriété individuelle de leurs actions, attribuées dans la pensée de tous au syndicat lui-même. Durant les années qui suivent, les deux coopératives lyonnaises maintiennent leurs activités avec plus ou moins de difficultés, pour faire face à la concurrence dans un environnement inadapté à leurs spécificités. Elles apparaissent comme un "refuge " pour certains salariés: syndiqués congédiés par un imprimeur suite à une action revendicative et trop zélés 
pour pouvoir espérer une autre embauche, "vieux travailleurs » et même " ouvriers peu sérieux ", selon les termes du conseil d'administration de l'INL. Les Lyonnais se font en outre défenseurs de la coopération au sein de la puissante Fédération du livre, lors du III Congrès typographique, en 1885: l'intervention des délégués de la section lyonnaise fait alors suite à une décision du comité central qui avait, d'autorité, supprimé le paragraphe relatif à l'idée d'une aide financière pour la fondation d'imprimerie coopérative. Cette résolution arbitraire est révélatrice du manque d'enthousiasme de la fédération pour les actions de type réformiste. Elle a pour contexte l'expérience malheureuse de la coopérative créée par le syndicat des typographes parisiens (et proche de la fédération), qui s'était soldée par une cessation d'activité pour activité déficitaire. La défense du principe coopératif et son rétablissement dans les statuts sont obtenus notamment grâce à l'argumentation des typographes lyonnais, illustrée par la réussite des coopératives de la ville.

\section{Le syndicat des typographes lyonnais et le modèle coopératif}

Pourtant, même à Lyon, le fonctionnement de la coopération ne fut pas toujours simple: l'histoire de l'INL et les modifications substantielles qui durent être apportées dans ses statuts le montrent. En 1888, le syndicat doit prendre la décision de rendre l'INL complètement indépendante en raison de ses difficultés financières. L'idée, âprement défendue en 1882, de considérer le syndicat comme personne morale seule propriétaire de la coopérative est abandonnée sous le prétexte que de nouveaux syndiqués inscrits depuis la cessation de l'impôt sur les salaires n'ont pas contribué à former le capital coopératif. En réalité, la décision est révélatrice de la désillusion des syndiqués face à une structure coopérative qui ne concrétise pas les espoirs de diminution des mouvements de grève et qui est progressivement vue comme un obstacle à la prospérité du syndicat, dont elle absorbe la plupart des ressources. Les deux entités sont alors séparées au terme d'une opération financière complexe, mais consensuelle. Cette scission n'est pas sans effet sur le fonctionnement de l'imprimerie. Outre qu'elle estompe une partie des divergences entre les deux coopératives de production de la typographie lyonnaise, elle bouleverse l'ordonnancement de l'INL, déjà peu florissante: le directeur, en fonction depuis la création, est remercié, et il achète une imprimerie qui fait une concurrence facile à l'INL; la direction de la structure pose alors problème, puisque pas moins de quatre directeurs se succèdent en moins de dix-huit mois; de plus, les statuts sont (modérément) modifiés en 1890, et le siège est transféré l'année suivante. En dépit de ces difficultés, l'INL parvient à maintenir ses activités et, à la fin des années 1890, elle occupe jusqu’à une quarantaine d'ouvriers (sociétaires et auxiliaires) dans un atelier social bénéficiaire. De 1894 à 1897, la structure reçoit également du ministère $\mathrm{du}$ Commerce une subvention de 5000 francs. 
Couvrant plus d'un siècle, les registres des délibérations du syndicat des typographes témoignent de la richesse des pratiques coopératives autant que de leurs ambiguïtés. Certes, la formule permet une implication constante de la classe ouvrière dans les choix de gestion et d'orientation des structures productives, mais, plus largement, ces expériences lyonnaises sont aussi révélatrices de l'ambivalence des rapports entre syndicats (notamment à leur création) et associations coopératives lorsque ces dernières deviennent autonomes. L'histoire des coopératives typographiques lyonnaises témoigne de cette confrontation et de la difficulté quotidienne pour leurs dirigeants de mener une action à la fois conforme aux valeurs qui animent la structure et viable sur le plan économique.

Ce conflit de valeurs fut particulièrement sensible lors de la formulation de revendications salariales ou à l'occasion de la survenance de conflits collectifs. Une illustration de ce type de dissension est donnée lors du conflit qui surgit au sein de l'INL en juin 1917, à l'occasion de discussions salariales. Cet exemple permet de mesurer à quel point l'imbrication de la coopérative et du syndicat rend délicates les relations entre équipe dirigeante et salariés. La difficulté survient ainsi lorsque les ouvriers typographes, donc syndiqués, de la coopérative demandent au comité directeur du syndicat un soutien pour que " l'indemnité de vie chère » des ouvriers papetiers, non syndiqués, soit accordée par le conseil d'administration de l'INL. La demande est alors ferme et assortie d'une menace de grève des syndiqués, par solidarité avec les papetiers, dont ils jugent les requêtes légitimes. Après de vives discussions, le comité du syndicat se pose en " médiateur " du conflit, afin de faciliter la recherche d'un terrain d'entente entre les parties. Il convoque le conseil d'administration de l'INL, qui s'exprime, lors d'une séance extraordinaire, par la voix de son délégué: il oppose à la demande une fin de non-recevoir en déclarant que les exigences sont de nature à mettre en péril le fonctionnement de la structure. Les discussions qui s'ensuivent montrent les pressions du syndicat et ses tentatives interventionnistes, à peine masquées, dans des conseils et des incitations très insistantes. Appelé par le syndicat à se réunir à nouveau, le conseil d'administration de l'INL accepte néanmoins de faire quelques concessions financières aux ouvriers, à la satisfaction du syndicat.

\section{Conclusion}

Les deux plus anciennes sociétés coopératives typographiques lyonnaises disparurent au cours des années 90, lorsque la technologie informatique révolutionna le design graphique et typographique. Leur fonctionnement est révélateur d'une histoire tumultueuse entre syndicats et coopératives. Une histoire faite de heurts, de rapprochements, de mises en doute et d'espoirs, selon les époques. En raison de leurs attaches syndicales très fortes, ces deux structures confrontèrent, sur le terrain, les militants 
syndicaux à des logiques auxquelles ils n'étaient pas accoutumés parce qu' ils étaient formés à la lutte contre un patronat de type capitaliste.

Cette illustration lyonnaise est une invitation plus large à réfléchir sur le défi supplémentaire que les syndicats doivent relever, au moment où ils soutiennent la solution de la coopération pour la reprise ou la transmission d'entreprise. Elle pose la question de l'invention d'une nouvelle logique d'action collective qui réponde aux besoins de salariés devenus associés et leur permette de s'affirmer au sein des Scop. 


\section{Bibliographie}

Frobert L., 2007, « Lyon, le Manchester français ", L'Histoire, n 320, mai.

Gueslin A., 1987, L'invention de l'économie sociale, Paris, Economica.

Lequin Y., 1977, Les ouvriers de la région lyonnaise (1848-1914), vol. 2, Les intérêts de classe et la République, Presses universitaires de Lyon, Imp. Chirat.

Moissonnier M., 1984, "Le mutuellisme lyonnais ", Prévenir, "Autour du premier congrès des sociétés de secours mutuels, Lyon, 1883-1983", mai 1984, p. 51-56.

Pierre de Vellefrey G. (de), 1875, « De la loi du 24 juillet 1867 ", thèse pour le doctorat, Paris, Imp. Donnaud.

Rude F., 1977, Le mouvement ouvrier à Lyon, Lyon, Fédérop.

Rude F., 2001, La révolte des canuts, 1831-1834, Paris, La Découverte.
Sheridan G., 1981, The social and economic foundation of association among the silk weavers of Lyon, 1852-1870, New York, Arno Press a New York Company.

Tchernoff I., 1905, Associations et sociétés secrètes sous la II République 1848-1851, Bibliothèque d'histoire contemporaine, Paris, Alcan.

Les documents suivants ont été consultés pour cette recherche:

-Archives du syndicat des typographes, institut départemental d'histoire CGT.

- Procès-verbaux des séances du comité de l'Annexe de la $31^{\mathrm{e}}$ société de secours mutuels des 15 et 23 juin 1862, 31 décembre 1866, 13 juin 1882, 5 août 1888, 5 juin 1917.

- Courriers du syndicat des 27 juin 1978, 12 et 15 juin 1979.

- Lettre ouverte aux adhérents du syndicat du 15 juin 1979.

- Archives municipales de Villeurbanne, sérieZ, divers 8Z, 1864-1999. 